Gynecologic and

Obstetric Investigation
DOI: $\underline{10.1159 / 000339972}$
Received: November 22, 2011

Accepted after revision: June 10, 2012

Published online: August 9, 2012

\title{
Surgical Treatment of Mixed and Urge Urinary Incontinence in Women
}

\author{
Wolfram Jäger Olga Mirenska Sabine Brügge \\ Urogynaecology Unit, Department of Obstetrics and Gynecology, University of Cologne, Cologne, Germany
}

\section{Key Words}

Pelvic surgery · Stress urinary incontinence - Urge urinary incontinence

\begin{abstract}
Background/Aims: The etiology of urge urinary incontinence is unknown. Pharmacological treatments are disappointing, since they are only slightly more effective than a placebo. In this study, we analyzed whether the surgical replacement of the uterosacral ligaments by an alloplastic tape can cure patients with mixed and urge urinary incontinence. Methods: This study was a nonrandomized clinical trial. The study was performed in the Department of Gynecology of a general hospital and a university clinic. 135 women with urge or mixed urinary incontinence were operated on. The utero-sacral ligaments as well as the pubo-urethral ligaments - if necessary - were replaced by alloplastic tapes. Clinical outcome was evaluated immediately after surgery, 3 months later and 1 year later. Outcome was classified as cure, improvement or failure and recurrences. Results: 102 patients (77\%) were cured and a further 24 patients (18\%) showed improvement after surgery. 19 patients developed a recurrence between 5 and 33 months after surgery. Conclusion: We therefore conclude that most patients with urge and mixed urinary incontinence can be cured by surgery.
\end{abstract}

Copyright $\odot 2012$ S. Karger AG, Basel
(C) 2012 S. Karger AG, Basel

0378-7346/12/0742-0157\$38.00/0

Fax +4161306 1234

E-Mail karger@karger.ch

www.karger.com
Accessible online at: www.karger.com/goi

\section{Introduction}

Urge urinary incontinence is a most bothersome disorder and affects probably every third postmenopausal woman. This triad of factors which it involves - urgency, frequency and incontinence - has detrimental effects on the patient's quality of life and proves to be a source of anxiety [1].

Urge urinary incontinence is considered to be related to incorrect detrusor muscle function or innervation. Pharmacological treatments are disappointing, since they are only slightly more effective than placebo [2].

In 1991, de Lancey [3] emphasized the importance of the support of the genital tract for continence function. He considered in that respect that the attachments of the ligaments at the cervix were so important that he proposed to perform supracervical hysterectomy instead of total hysterectomy whenever possible.

This suggested critical role of the supportive structures for incontinence and especially for urge urinary incontinence is indicated by observations in patients with descensus uteri. Up to $50 \%$ of these patients suffer from urge urinary incontinence $[4,5]$. Descensus uteri is not a disease of the uterus, so it is not surprising that neither vaginal nor abdominal hysterectomy can cure urge urinary incontinence in these patients [6]. 
In his 'Integral Theory', Petros [7] pointed out the importance of the posterior compartment for establishing continence function. He assumed that the uterosacral ligaments (USL) are particularly essential for causing urge urinary incontinence.

We therefore hypothesized that the association between descensus uteri and urge urinary incontinence could be caused by a defective functioning of the USL.

In 2004, we therefore decided to repair the USL in these patients instead of removing the uterus [8]. Since the USL in postmenopausal patients do not have a ligamentous structure but are more or less a peritoneal fold with only microscopically detectable musculature, a classical repair was not possible. We therefore replaced it with alloplastic tapes.

Since all of our patients were past child-bearing age, we performed a supracervical hysterectomy and sutured the tapes on the cervical stump where we could cover them with peritoneum.

We soon found out that our operation, which we called 'cervical-rectal-sacral fixation' (CERESA), could cure patients with urge urinary incontinence. At first we refused to operate on hysterectomized women since we thought that the cervix and the surrounding nerves would be important for the success of our surgery. However, patients with vaginal vault prolapse and urge urinary incontinence also asked for that operation. On these patients we performed essentially the same surgery: the only difference was the placement of the tapes at the vaginal vault instead of the cervix. This vaginal-rectal-sacral fixation (VARESA) also led to cure in 7 of the first 10 hysterectomized women with urge urinary incontinence [8].

Following the recommendations of our Ethical Committee, we then observed a moratorium of one year in order to find out any long-term side effects. Within 1 year 2 of the 13 previously cured patients developed a recurrence and 6 patients became stress incontinent ('de novo stress'). However, these patients were then cured by the placement of a transobturator tape (TOT).

Despite the recurrence rate of $25 \%$ and a 'de novo stress' rate of $50 \%$, an increasing number of women opted for VARESA or CERESA, respectively. After intensive discussions we finally decided to start a study in which we offered VARESA or CERESA to all patients with urge and mixed incontinence who had failed conservative treatment.

We now report the results of the first 135 patients treated with VARESA and CERESA.
Table 1. Different categories of incontinence

Category 0 was always defined as: no problems with early urine loss or frequency of voiding ('I have no problems with voiding! I can do anything I like without any limitations').

Holding categories:

Category 1: patients who can hold the urine more than $3 \mathrm{~min}$ but less than $10 \mathrm{~min}$ ('When I go shopping and feel the need to urinate I must be in the toilet within few minutes').

Category 2: Patients who could hold the urine for less than 3 min ('When I feel the need to urinate I must be in the toilet within a minute!' or 'I always loose urine, I cannot hold it at all').

Frequency of voiding categories:

Category 0: less than 8 times per day

Category 1: between 8 and 15 times per day

Category 2: more than 15 times per day ('During the day I go to the bathroom at least two times per hour').

Stress incontinence was not further categorized for the purpose of that study.

Cure was defined as absence of all subjective and objective incontinence symptoms (category 0). Improvement was defined as a lower category as before treatment.

\section{Material and Methods}

The study was initiated in the Department of Gynecology in a general hospital (EVK Dortmund) and continued at the Department of OB/GYN at the University of Cologne. It got the institutional study name 'VARESA II'. It was approved by the Ethical Committee of the general hospital (EL13-2007).

Diagnosis of urge or stress urinary incontinence was based on the subjective complaints of the patients $[9,10]$.

Urodynamic measurements were intended in every patient before surgery and at the end of the study. Urodynamic measurements were a standard procedure during VARESA I. We produced an enormous amount of data but the final question was only whether the patient was cured or not. The patient's subjective feeling was important, rather than her objective measurements. In accordance with recommendations in the literature, we therefore decided that the subjective feeling of the patient was the primary aim of the study [10].

We used the PETROS questionnaire. However, it turned out that all patients were suffering from severe incontinence and that further subclassifications (e.g. volume of urine loss) were unnecessary. This was also based on the observation that after surgery patients were usually cured or not. Based on the recommendations of the statistician, we therefore reduced our questionnaire to 19 questions with three main categories. Since all patients came to our institution either because of their voiding frequency or their problem of reaching a toilet in time, we roughly subdivided urge urinary incontinence between their main problems of 'frequency' and 'holding' [10]. However, each patient was counted in both categories. Stress incontinence was the third category [9]. Each patient was categorized according to these findings and to the variables shown in table 1 . 
Previous experiences showed that all patients who came to our institution were pre-treated (data not shown) and did not accept a randomization to a non-surgical treatment arm. We therefore decided to perform an observational study in patients with mixed or urge urinary incontinence who had not been operated on before because of incontinence or prolapse. The study was explained in detail to all patients. They were informed about the experimental character of the study. We reported an estimated rate for improvement of $50 \%$ for the whole study population and a recurrence rate of $25 \%$ and a $50 \%$ rate of 'de novo' stress incontinence after surgery. In such an instance they would get a second operation (TOT). No prediction for the individual's outcome was made. Whenever possible these explanations were given in presence of proxies (husband or family members).

According to the study protocol, the effects of surgery were evaluated in each patient 4 weeks after surgery. Intermediate controls were scheduled after the 10th, 30th and 60th patient or after 6 and 12 months. The study would be stopped if the cure or improvement rate was less than $50 \%$ of the operated patients. The duration of the study was planned for 2 years with an observation period of 1 year after surgery. The study started on 1 March 2007 and was finished on 28 February, 2009. The final interviews of all patients took place from March to September 2010, giving an observation period of between 12 and 41 months (median 22 months). All patients gave informed written consent.

\section{Study Protocol}

Only patients with the diagnosis of mixed and urge urinary incontinence were eligible for the study. Patients with stress urinary incontinence only were excluded. Patients who complained of a high frequency of voiding even during the night (more than one time per $2 \mathrm{~h}$ of sleep) but who were, however, otherwise continent, were excluded from the study. Since we hypothesized another etiology of this particular disorder, such patients were enrolled in another study.

Patients who had already been operated on with the intention of improving incontinence (e.g. vaginal sling operation, colporraphy, colpo-suspension) or who had had any other kind of prolapse surgery were excluded.

Diagnosis was established upon the complaints of the patients using the questionnaire mentioned above [11]. Urogynecological examinations were performed in every patient. A smear was obtained from the cervix or the vaginal vault to exclude neoplastic disorders. A vaginal ultrasound examination was performed to detect irregularities of the ovaries or other parts of the small pelvis. A cough and a pad test were done with full bladder, and urine was tested for irregular findings.

All patients received antibiotic treatment for 3 days starting on the day of surgery. All operations were performed by the first author (W.J.). Patients were re-examined 4 weeks after surgery. Basically, all examinations which were done before surgery were repeated. If a patient developed stress urinary incontinence ('de novo stress'), a TOT was performed. In these patients a clinical re-examination was performed 3 months after TOT.

Interviews were performed by gynecologists of our unit (O.M., S.B.) at the 4 weeks after surgery visit and every 6 months thereafter by telephone. If patients had any complaints, they were invited to the clinic for another clinical examination. All first interviews and gynecological examinations were done by the first author (W.J.).

Surgical Cure of Urge Urinary

Incontinence

\section{Operations}

The aim of VARESA and CERESA was to replace the USL as exactly as possible. Both operations were done by laparotomy. As a first step, the USL were identified, either directly from the beginning at the cervix or by stretching the vaginal cuff with a probe placed in the vagina. In CERESA a supracervical hysterectomy was performed as the first step. Within the first centimeters from the cervical ring the USL have a fibrous structure, while thereafter they appear as a peritoneal fold with no visible structural elements. The folds were either detected by extending the uterus or the vaginal vault. They were incised and from that incision the nervus hypogastricus was identified. Then the tissue above the sacral vertebrae 1 and 2 was incised and put aside until the periost was visible. Thereafter, the alloplastic tapes were placed with two nonresorbable sutures at the periost on both sides of the rectum $[8,12]$. The size of the tapes was $1.5 \times 13 \mathrm{~cm}$ (DynaMesh, FEG, Aachen, Germany). The tapes were then placed in the incised and prepared folds (USL). In CERESA the end of the tapes were fixed at the surface of the cervical stump. The bladder remained on the cervix. In already hysterectomized patients, a probe was placed in the vagina. In these patients the bladder remained on the vagina and the tapes were sutured at the vaginal vault. The tapes were shortened and the peritoneum of the bladder and the posterior vagina was closed above the fixation points. The rectum was fixed to the tapes in the fold with two resorbable sutures $3 \times 0$ placed at the mesorectum.

Those patients who had a mixed urinary incontinence or developed a 'de-novo' stress urinary incontinence received a TOT according to the method of Delorme [13].

\section{Statistical Analysis}

Pertinent clinical data, e.g. age, body weight, number of previous treatments, number of vaginal births, were also analyzed for prediction of treatment failure.

Between-group comparisons for categorical variables were performed using $\chi^{2}$ or Fisher's exact tests. Pre- and post-scores were compared using the Wilcoxon test and between-group comparisons of scores were performed using Mann-Whitney tests. For the influence of clinical data multivariate logistic regression analyses were performed. All analyses were performed using SPSS version 17 (SPSS, Chicago, Ill., USA) at a significance level of 5\%.

\section{Results}

135 patients were treated in the study. One patient suffered from a stroke 4 weeks after VARESA and 1 patient developed Alzheimer's disease during follow-up and could therefore not be evaluated. Therefore, the analysis is based on the data of the remaining 133 patients (fig. 1).

Based on the symptoms, 64 patients (48\%) suffered from mixed urinary incontinence and 69 patients (52\%) had urge urinary incontinence. 114 patients had severe holding problems and 91 patients went to the toilet more than 8 times per day (table 2). No patient had a uterine or vaginal prolapse grade 4 . Two patients had a descensus grade 3 and 2 patients had grade 2 [6]. 
Fig. 1. Distribution and outcome of patients in the VARESA II study. Note that 20 patients with mixed incontinence and 22 patients with urge incontinence were cured without a TOT. 91 patients needed a TOT.

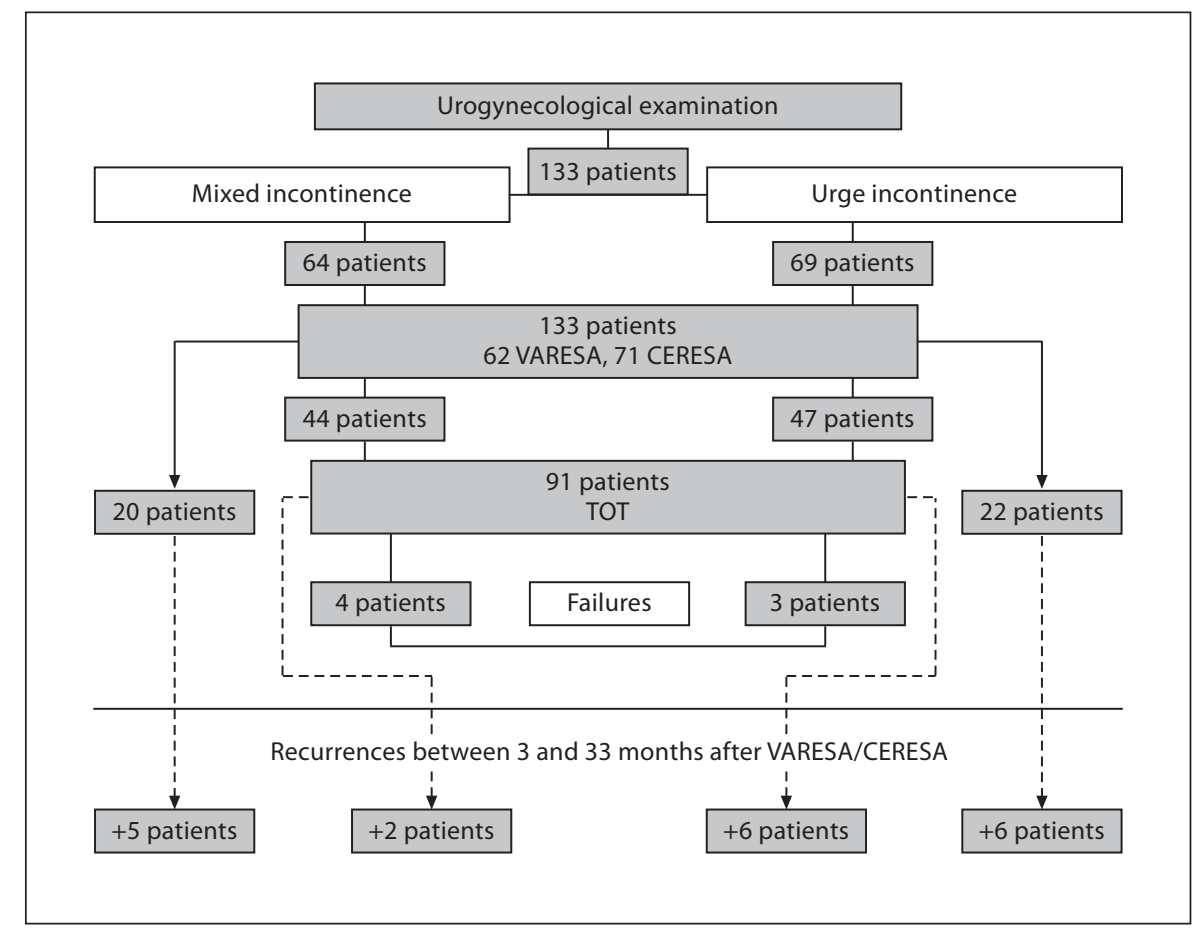

Table 2. Distribution of the incontinence symptoms of all 133 patients according to the type of surgery (VARESA or CERESA)

\begin{tabular}{|c|c|c|c|c|c|c|}
\hline \multirow{2}{*}{$\begin{array}{l}\text { Operation: } \\
\text { Number of patients: } \\
\text { Mean age }( \pm 2 \mathrm{SD}) \text { : }\end{array}$} & \multicolumn{3}{|c|}{ VARESA } & \multicolumn{3}{|c|}{ CERESA } \\
\hline & \multirow{2}{*}{\multicolumn{3}{|c|}{$\begin{array}{l}66 \\
62(49-75)\end{array}$}} & \multirow{2}{*}{\multicolumn{3}{|c|}{$\begin{array}{l}67 \\
65(51-79)\end{array}$}} \\
\hline Mean age $( \pm 2 \mathrm{SD})$ : & & & & & & \\
\hline & before & after & $\mathrm{p}$ & before & after & $\mathrm{p}$ \\
\hline \multicolumn{7}{|l|}{ Stress } \\
\hline No & 32 & 58 & $<0.05$ & 37 & 60 & $<0.05$ \\
\hline Yes & 34 & 8 & $<0.01$ & 30 & 7 & $<0.01$ \\
\hline \multicolumn{7}{|l|}{ Holding } \\
\hline No problem & 9 & 44 & $<0.01$ & 10 & 50 & $<0.01$ \\
\hline Longer than 3 min but less than $10 \mathrm{~min}$ & 37 & 18 & $<0.05$ & 35 & 16 & $<0.05$ \\
\hline Less than $3 \mathrm{~min}$ & 20 & 4 & $<0.01$ & 22 & 1 & $<0.01$ \\
\hline \multicolumn{7}{|l|}{ Frequency } \\
\hline No problem & 22 & 44 & $<0.01$ & 20 & 60 & $<0.01$ \\
\hline Between 8 and 15 times per day & 19 & 10 & $<0.05$ & 25 & 6 & $\mathrm{~s}<0.01$ \\
\hline More than 15 times per day & 25 & 2 & $<0.01$ & 22 & 1 & $<0.01$ \\
\hline
\end{tabular}

Each patient is categorized according to her stress, holding and frequency symptoms. Number and mean age $(95 \% \pm 2$ SD) of patients and change of symptoms before and after the respective surgery including TOT. Categories of symptoms according to table 1 . 


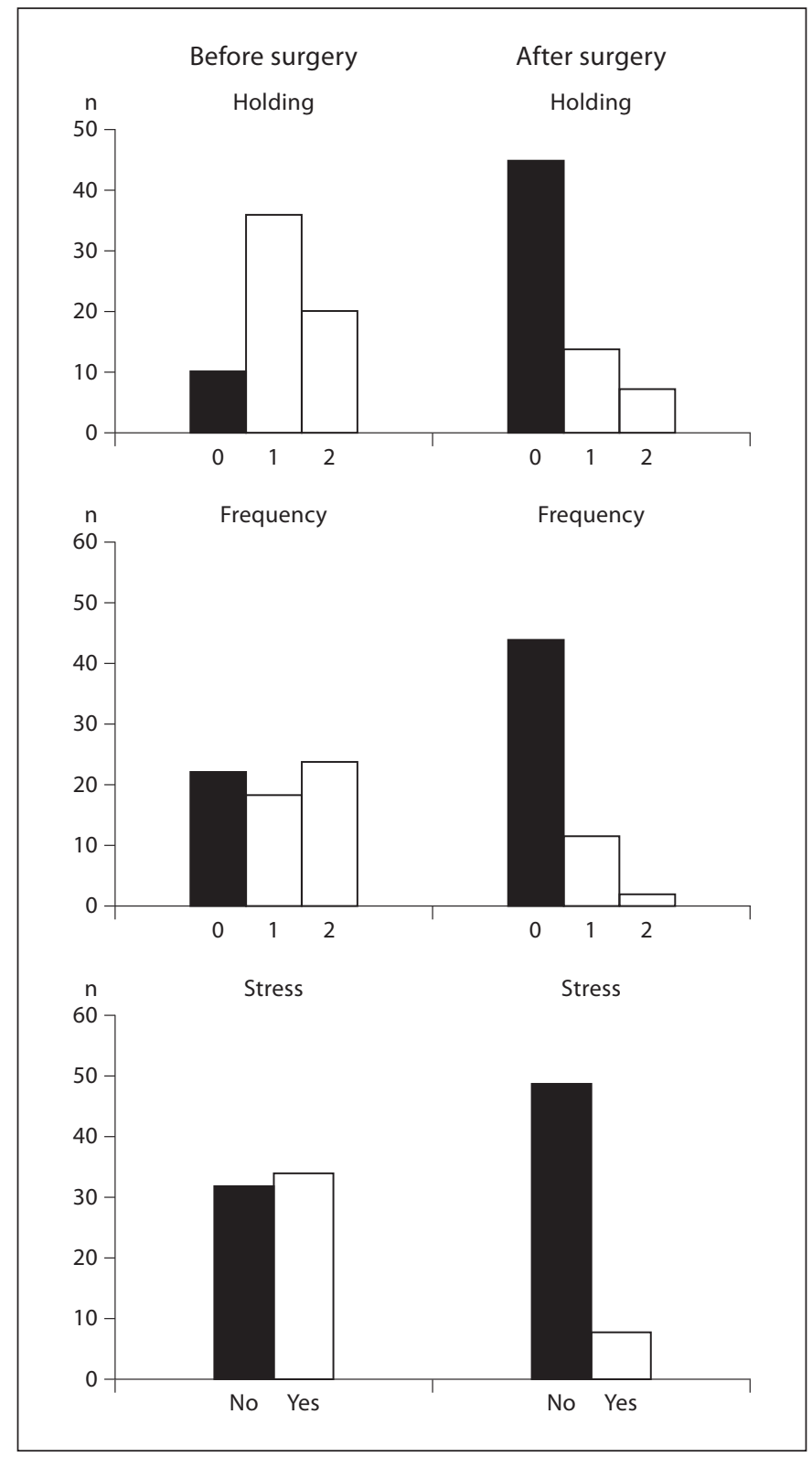

Fig. 2. Distribution of symptoms before (left side) and after (right side) VARESA. Shown are the numbers of patients according to the symptom categories. The categories $(0,1$ and 2$)$ were defined as follows: category $0=$ no problems with early urine loss or frequency of voiding. Holding (first row of columns): category $1=$ can hold the urine for more than 3 min but less than $10 \mathrm{~min}$; category 2 = can hold the urine for less than $3 \mathrm{~min}$. Frequency (second row of columns): category $1=$ between 8 and 15 voiding episodes per day; category 2 = more than 15 voiding episodes per day. Every patient was categorized in each of the different forms of incontinence.

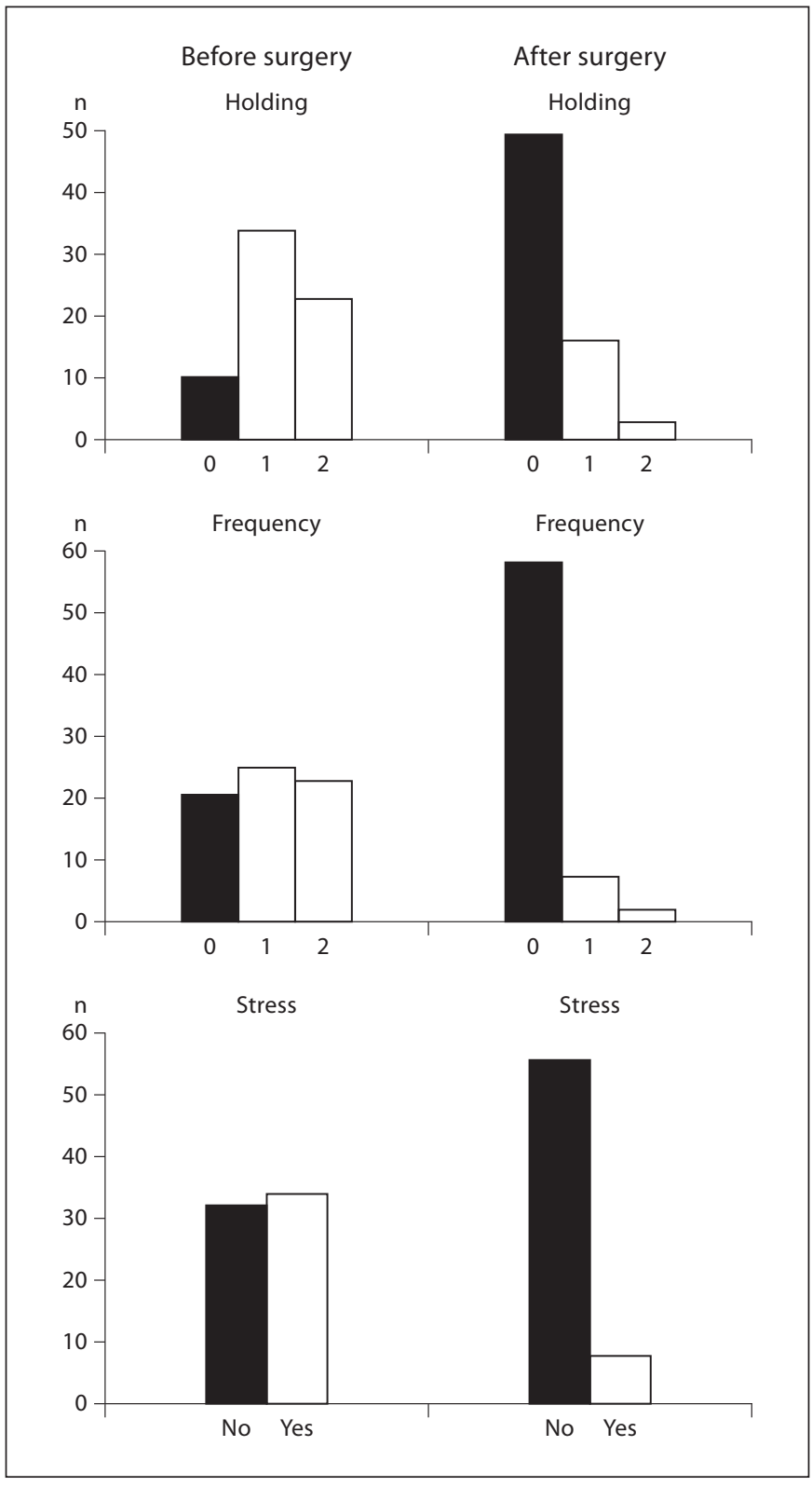

Fig. 3. Distribution of symptoms before (left) and after (right) CERESA. Shown are the numbers of patients according to the symptom categories. The categories $(0,1$ and 2$)$ were defined as follows: category $0=$ no problems with early urine loss or frequency of voiding. Holding (first row of columns): category $1=$ can hold the urine for more than $3 \mathrm{~min}$ but less than $10 \mathrm{~min}$; category 2 = can hold the urine for less than $3 \mathrm{~min}$. Frequency (second row of columns): category 1 = between 8 and 15 voiding episodes per day; category $2=$ more than 15 voiding episodes per day. Every patient was categorized in each of the different forms of incontinence. 
Table 3. Distribution of all 133 patients according to type of incontinence and surgery (VARESA or CERESA) and outcome at the end of the study

\begin{tabular}{lrrrl}
\hline & VARESA & CERESA & Total & $\mathrm{p}$ \\
\hline Mixed & 34 & 30 & 64 & \\
$\quad$ Cured & 26 & 23 & 49 & n.s. \\
Improved & 6 & 5 & 11 & n.s. \\
Failed & 2 & 2 & 4 & n.s. \\
Urgency & 32 & 37 & 69 & \\
Cured & 26 & 27 & 53 & n.s. \\
Improved & 4 & 9 & 13 & n.s. \\
Failed & 2 & 1 & 3 & n.s. \\
\hline
\end{tabular}

$\mathrm{p}$ values for comparison of both treatments were not significant (n.s.).

66 patients (49\%) were operated on by VARESA and 67 patients (51\%) by CERESA. The operations lasted between 58 and $145 \mathrm{~min}$, with a mean of $85 \mathrm{~min}$. The mean blood loss was less than $50 \mathrm{ml}$. However, in 2 patients with a bleeding of the sacral venous plexus the blood loss was 1.2 and 1.5 liters. These patients were not counted during the statistical analysis for operation time and blood loss. Mean age of the patients was 62 years in VARESA $( \pm 13$ years) and 65 years in CERESA ( \pm 14 years). The outcome after surgery was not statistically different between VARESA and CERESA (fig. 2, 3).

91 patients $(68 \%)$ developed a stress urinary incontinence within 3 months of primary surgery and got a TOT. All were continent thereafter.

42 patients $(32 \%)$ were cured without a TOT. This group of 42 patients consisted of 22 patients with urge and 20 patients with mixed urine incontinence.

Patients with different types of incontinence were nearly equally distributed in both treatment groups (table 3). 49 patients (76\%) with mixed urinary incontinence were cured and 11 patients (17\%) improved. In 4 patients (7\%) no beneficial effect of surgery was detected. 53 patients $(77 \%)$ with urge urinary incontinence were cured and 13 patients (19\%) were improved (table 3 ).

The overall cure rate was $77 \%$ (102 of 133 patients). 24 patients (18\%) reported a subjective improvement after surgery.

In 7 patients (5\%) no cure or improvement was achieved. Furthermore, 19 patients (15\%) of the cured or improved patients developed a recurrence of their primary symptoms between 4 and 12 months after surgery (fig. 1). They all reported that the recurrence was not a slow progress but came suddenly. Eight of these patients had developed a stress urinary incontinence and were cured after a TOT. 5 of the remaining patients with a recurrence of the urge symptoms agreed to a second-look laparoscopy/laparotomy. In these patients we found a disruption of the tape at the side of the vaginal vault ( 3 patients) or the cervix (2 patients). After refixation, patients were continent again. The remaining 6 patients refused second look operations, so the reason for recurrence could not be evaluated.

According to statistical analysis, no risk factors for recurrence could be defined. It was also not possible to predict which patients could not be cured.

\section{Discussion}

Since the etiology of urge urinary incontinence is unknown, the definition of this disorder is based on symptoms. The expression of symptoms varies considerably from patient to patient. Furthermore, the same symptom has different individual perceptions. This has led to an increasing number of questionnaires and questions. Yet in spite of this, treatment options remain sparse [14-16].

In our VARESA I study, we had already realized that urodynamic measurements were not helpful. The patient knew by herself if she lost urine during coughing or sneezing and her urgency problems were always the same: either with the frequency of voiding, or with the problem of reaching the toilet in time. Since all our patients were suffering and already had a considerable number of medical pretreatments, they asked for surgery. Therefore, our VARESA II study was not based on the results of the urodynamic measurements but on subjective symptoms.

One could argue that the high cure rate of $77 \%$ in our study for a disease previously considered to be incurable could not be true and must be based on a priori misclassification of the patients.

However, all the patients came to our institution only because of their massive problems with urge urinary incontinence. More than $70 \%$ of the patients categorized their personal incontinence situation as the worst quality of life. $32 \%$ of these patients were wet all the time or could suppress voiding only for seconds. Another $35 \%$ of the patients went to the toilet more than 15 times per day and some of them every $30 \mathrm{~min}$. These patients refused any further conservative treatment since no previous treatment had helped them. Since the results of the study were not published, all patients came because of the recommendations of the cured patients. That was probably the best confirmation of the results by itself. 
The results of our study thus showed that urge and mixed urinary incontinence can be cured by surgery, at least as a proof of the principle.

Some of the findings were surprising for us. We expected that patients with mixed urinary incontinence would definitively need a TOT [15]. For organizational reasons we decided not to perform the TOT in this study during VARESA or CERESA. Surprisingly, it turned out that one-third of these patients did not have stress symptoms anymore after VARESA or CERESA. However, 1 year after the end of the study, 5 of these 20 patients nevertheless became stress incontinent again.

On the other hand, two-thirds of the patients with urge incontinence developed stress incontinence immediately after VARESA or CERESA ('de novo stress'). 4 more patients became stress incontinent after the end of the study within the next 12 months.

We have no explanation for these findings. The observation that $80 \%$ of patients with mixed or urge urinary incontinence needed repair of both ligaments, i.e. the USL and the pubourethral ligaments, led us to assume that continence could probably only be achieved by the correct function of both structures [18]. This assumption was further substantiated by the observation that most of the 'totally wet' patients only became 'dry' when they got the TOT. That would nicely fit with the 'bridge allegory' of Petros and Woodman [19], where continence is based on the correct function of the 'tows on both pylons'.

When we removed the trans-urethral catheter after surgery, patients were either cured or not. According to our protocol, the results were based on the examination 4 weeks later. In 7 patients, no improvement was noted. 24 patients reported an improvement of their symptoms. However, these heavily suffering patients may have reported small changes as improvement. As they were not cured, we counted them as treatment failures. Analyzing all the clinical data of the patients, we could not find any discriminating factor or a combination of factors which would predict the outcome of surgery in terms of cure. So we have no explanation for the $23 \%$ failure rate.

Stress urinary incontinence can be cured by TVT or TOT. This study demonstrates for the first time that urge urinary incontinence can also be cured by surgery. The failure rate, however, demonstrates that some more problems still need to be solved. All steps of VARESA and CERESA operations were standardized. The operations were subdivided into several steps and analyzed. No major differences were found. The only part of the operation which was not clearly defined was the 'tension-free' vaginal tape. Referring to the bridge allegory, it could mean that the tension of the tows of the front pylon (the TOT) must be balanced with the tows on the back pylon. Furthermore, it cannot be excluded that an etiologically different group of patients, e.g. those with psychosomatic disorders, were not detected by our inclusion criteria, i.e. our questions. Perhaps we have to find the right questions for the right problems.

Further carefully designed clinical studies should focus on these different aspects. Specialized clinical units must solve these problems before the operation becomes general practice. Then there will be a great chance of achieving a cure for all patients suffering from stress and urge urinary incontinence.

\section{Acknowledgements}

We thank C. Cetin and S. Saria for their assistance during surgery. We are grateful to E. Neumann and M. Kortmann for their technical support and organization of the study. We further appreciated the help of A.C.J. Jäger for statistical analysis of the data. We thank P. Petros in the Popperian sense for the Integral Theory without which this study would not have been possible.

\section{References}

1 Nygaard I: Idiopathic Urge Urinary Incontinence. NEJM 2010;363:1156-1162.

-2 Shamliyan TA, Kane RL, Wyman J, Wilt TJ: Systematic review: randomized, controlled trials of nonsurgical treatments for urinary incontinence in women. Ann Intern Med 2008; $148: 459-473$.

3 de Lancey JOL: Structural support of the urethra as it relates to stress urinary incontinence: the hammock hypothesis. Am J Obstet Gynecol 1994;170:1713-1723.
4 Lakeman MME, van der Vaart CH, Roovers JP: Hysterectomy and lower urinary tract symptoms: a nonrandomized comparison of vaginal and abdominal hysterectomy. Gynecol Obstet Invest 2010;70:100-106.

5 Van Brummen HJ, van de Pol G, Aalders CIM, Heintz APM, van der Vaart CH: sacrospinous hysteropexy compared to vaginal hysterectomy as primary surgical treatment for a descensus uteri: effects on urinary symptoms. Int Urogynecol J 2003;14:350355.
-6 Gustafsson C, Ekstrom A, Brismar S, Altman D: Urinary incontinence after hysterectomy - three-year observation study. Urology 2006;68:769-774.

7 Petros PE, Ulmsten UI: An integral theory and its method for the diagnosis and management of female urinary incontinence. Scand J Urol Nephrol 1993;(suppl 153):193.

8 Jäger W: Die operative Behandlung der Dranginkontinenz. Gyn 2010,15:400-404. 
9 De Wachter S, Wyndaele JJ: Frequency-volume charts: a tool to evaluate bladder sensation. Neurourol Urodyn 2003;22:638-642.

10 Blaivas JG, Pnanaggopoulos G, Weiss JP, Somaroo C: The Urgency Perception Score: validation and test-retest. J Urol 2007;177: 199-202.

11 Petros PEP: The Female Pelvic Floor: Function, Dysfunction and Management According to the Integral Theory. Berlin, Springer, 2004, p 58.

13 Delorme E: Transobturator urethral suspension: mini-invasive procedure in the treatment of stress urinary incontinence in women. Prog Urol 2004;11:1306-1313.

14 Coyne KS, Matza LS, Thompson CL: The responsiveness of the Overactive Bladder Questionnaire (OAB-q). Qual Life Res 2005; 14:849-855.

15 Cardozo L, Coyne KS, Versi E: Validation of the urgency perception scale. BJU Int 2005; 95:591-596.

12 Bellfin JM, Contreras LA, Bujan J, CarreaSan MA: Experimental assay of a dual mesh polytetrafluoroethylene prosthesis (non-porous on one side) in the repair of abdominal wall defects. Biomaterials 1996;17:23672372.
16 Nixon A, Colman S, Sabounjian L, Sandage B, Schwiderski UE, Staskin DR, Zinner M: A validated patient reported measure of urinary urgency severity in overactive bladder for use in clinical trials. J Urol 2005; 174:604607.
17 Minassian VA, Stewart WF, Hirsch AG: Why do stress and urge incontinence co-occur much more often than expected? Int Urogynecol J 2008; 19:1429-1440.

18 Ward KL, Hilton P: A prospective multicenter randomized trial of tension-free vaginal tape and colposuspension for primary urodynamic stress incontinence: two year follow-up. Am J Obstet Gynecol 2004;190: 324-331.

19 Petros PEP, Woodman PJ: The integral theory of continence. Int Urogynecol J 2008;19: $35-40$. 